to divide the phylum into sub-phyla, orders, classes, families and genera.

The recommendations of the provisional Committee of Nomenclature were recently presented for discussion at a well-attended meeting of the Virus Group of the Society for General Microbiology held in London. That the proposals received considerable criticism is perhaps not surprising in view of the strong and even emotional feelings usually generated by matters of taxonomy. Critics fell largely into three groups:

(a) Those who thought that at our present state of ignorance viruses cannot be classified on a rational basis and therefore no attempts should be made to devise a uniform system of nomenclature until more information. becomes available.

(b) A more important group of speakers pointed out a number of inaccuracies of fact and interpretation contained in the proposals and criticized the choice and hierarchical arrangement of criteria adopted for the classification system.

(c) Last, but not least, a number of speakers objected to the use of Latin names and to the adoption of a classification based on a fixed set of characters arranged in an arbitrary hierarchy. This group, represented mainly by plant virologists but supported by some eminent animal virologists, offered as an alternative a system based on Adansonian principles in which viruses would be designated by vernacular trivial names followed by a cryptogram containing information available or not on a number of unweighted characters (Gibbs et al., Nature, 209,$450 ; 1966$ )

It is difficult to imagine a system of virus nomenclature which will please all virologists, but it is hoped that discussions such as the one reported here will lead to proposals acceptable by a sizable majority.

H. G. Pereira

\title{
PROBABLE MODE OF LANDING OF LUNA 9
}

\author{
By Prof. T. GOLD \\ Center for Radiophysics and Space Research, Cornell University, Ithaca, New York
}

$\mathrm{F}$ ROM the data available through the news media concerning Luna 9 one can make some guesses as to its mode of landing which are of interest in a discussion of the lunar surface that is implied. Apparently, the spacecraft consisted of two parts: a bus of about $1 \cdot 5$ tons and a 2 -ft. diameter sphere of $220 \mathrm{lb}$. It would seem that the sphere had no propulsion or attitude control units aboard but was merely the container for the experimental equipment. The bus contained all the proximity radar, the propulsion and attitude control units and all the associated electronics required to achieve the soft landing; that is, the low velocity relative to the lunar surface. The sphere was separated from the bus when its propulsion and control function was over-that is, in the final free-fall period.

Such a technique has several advantages in simplicity over one where the entire vehicle lowers itself on to the surface to stand erect for the purpose of the experimentation and telemetry:

(1) Cancellation of the horizontal component of velocity. In the Luna 9 technique it will be permissible, and possibly even advantageous, for the sphere to approach the surface with a horizontal component of velocity several times larger than the vertical. The radar control of the landing thus avoids the necessity of high precision in the detection and cancellation of this component, which is one that must be more difficult to control than the vertical One supposes, therefore, that experiments will have been carried out of dropping the sphere in various ways on a variety of surfaces to decide what the permissible tolerance in the two components of velocity would be. On most surfaces the horizontal components of velocity could be between five and ten times greater than the vertical for a given permissible amount of damage or a given value of peak deceleration. One has to suppose, therefore, that the tolerances on the landing mechanism of the bus were such as to leave the sphere finally at impact with five to ten times as much horizontal as vertical velocity. We have no knowledge what the total velocity was beyond the statement that it was 'several metres per second'.

(2) Absence of attitude control. No attitude control is required in the final phase of the descent when all the active manoeuvre of the bus is finished. The sphere can be separated in some arbitrary way to skip, bounce and roll over the surface and finally come to rest. Its internal weight distribution may be such as to favour the desired way up and the subsequent opening of its four petals will assure that no extremely skew position can be maintained. It is obviously very unlikely that the sphere would lie precisely upside-down when the petals open so that they would fail to erect it.

(3) Freedom from sinkage. Such a mothod of landing will have appeared attractive in view of the unknown strength of the lunar surface. The loading should clearly be greatly reduced by abandoning all heavy rocket and control equipment and relying on a comparatively light sphere of a diameter comparable perhaps to that of the bus. Secondly, a large horizontal velocity at the impact of the sphere would tend to make the initial impact one of skipping like a stone on water, rather than one in which digging-in could occur. For the velocity tolerances that would be likely, and for as flat an angle of approach as given by the ratio of horizontal to vertical velocity of between five and ten, such a sphere would indeed skip, even on water, and certainly on most suggested types of lunar surface. The place where the sphere finally comes to rest in this mode will not have suffered any impact loading, and the compaction will only be that appropriate to the lunar weight of the sphere, which it would seem is $40 \mathrm{lb}$.

If indeed this were the mode of landing of Luna 9 then it would be clear why no impact information has become available that reflects on the strength of the lunar surface. The sphere has no attitude control and probably no internal knowledge of attitude in its tumbling phase. Even if accelerations were available for telemetry no meaningful integration of the accelerations could be carried out beyond gaining some idea of the length of time for which the sphere skipped and rolled. The small effect on the vertical component of acceleration which any compressibility of the surface might have made can then obviously not be sorted out when there is no independent directional information available in that phase. It is thus probable that nothing more can be said than that $a$ 2-ft. sphere pressing on the surface with a force of $40 \mathrm{lb}$. did not sink in more than a few inches. It is not quite clear what depth of sinkage the television pictures would have revealed but possibly as much as $6 \mathrm{in}$. would have gone unnoticed. The bearing pressure is then as low as 0.12 lb. per in. ${ }^{2}$. Most proposed types of lunar surface material would maintain that.

The bus is not visible in the panoramic view obtained by Luna 9. The panorama unfortunately shows the near ground over less than half the way around only because the vehicle did not in fact erect itself sufficiently accurately. 
The track that the sphere made on the surface would be likely to be from the direction of the bus and that track is thus also not on the available panorama. The other possibility, that the bus is not visible because it sank out of sight, but that on the other hand on the same surface the sphere left no visible track, is elearly most unlikely. The experimentation initially proposed then probably included a study of the visible track, and it was only the unfortunate angle in which the sphere came to rest that made this impossible.

The subsequent movement of Luna 9. It appears that between the picture-taking sequences, a movement occurred of unknown origin in which the camera position was changed by several inches. It was suggested in the Press that the experimenters involved had no knowledge of the origin of this movement and did not attribute it to any activity within the vehicle.

Earthquakes are clearly outside the range of reasonable explanations since a degree of such activity would be required that would have shown up in other ways.

On any dry, terrestrial ground, and in the absence of wind, it would seem very improbable that an object after coming to rest would make significant movements in a period of days. The observation must thus be taken to be a very significant one that implies properties of the lunar surface quite unlike those of terrestrial surfaces.

In whatever position the vehicle came to rest and whatever stresses, such as by spring-loaded panels, were then in effect, it would be necessary to discover some process with a time constant in the range of hours, to allow the movement to be triggered off. The immediate dynamical time constants of the settling would be in the neighbour- hood of seconds only. Plastic time constants in dry rock are many orders of magnitude too long. Thermal heating and cooling could have the right time constants, but the displacements resulting are so small that one would need to suppose the vehicle to be extremely delicately poised for such effects to trigger off a substantial movement.

It is thus quite a puzzle to discover some effect that would have been going on for a period of hours after the landing which, when it reached a certain stage, allowed a sudden resettling of the vehicle. Time constants of the same nature as those in an hour-glass may provide an explanation. If the material of the lunar ground were composed of small rock particles, slightly adhering to each other, it would be possible for there to be a lot of sub-surface hollow spaces. In particular, many small secondary impacts at rifle bullet speeds would make long tunnels at all angles through such a material. The structure that results would be the statistical equilibrium between the compacting effects of some types of impact and the tunnel-digging effects of others. On such a surface it would then not be too unlikely for the added weight of the vehicle to result in many little slides and collapses in internal cavities, which gradually reduce the strength of the ground and eventually let it settle. The time constants, as in an hour-glass, would be those of the dynamical time constants of falling grains but multiplied by the number of grains that had to fall in sequence to produce the effect.

While this explanation can be taken as a suggestion only, the fact that the ground tends to give way after some period of time under an additional load has probably to be taken seriously.

\section{SIGNIFICANCE OF INCLUSIONS IN CARBONACEOUS METEORITES}

$\mathrm{T}$ HE possibility has frequently been suggested and discussed that all meteorites which have become available for observation have originated from the same parent body, or from a comparable set of parent bodies, of asteroid dimensions. Such a theory is supported by hitherto observed instances of interbrecciations between: siderite-pallasite; siderite-mesosiderite; siderite-chondrite; siderite-achondrite; mesosiderite-chondrite; mesosiderite-achondrite; chondrite-achondrite (for a summary see refs. 1 and 2 ).

That such a common origin exists has not yet been proved through actually observed associations of those meteorites which could be included in the 'main carbonrich groups', with more than 0.2 per cent carbon (namely, $(A)$ carbonaceous chondrites; $(B)$ ureilites; $(C)$ enstatite chondrites).

The thirty or so carbonaceous chondrites which have so far been recorded have been sub-divided by Wiik ${ }^{3}$ into three types; the carbon + water + sulphur ranging between 24 and 30 per cent for 'type I'; 12 and 24 per cent for 'type II'; 3 and 12 per cent for 'type III'. Recent investigations have shown ${ }^{4}$ that 'type $I$ ' stones consist of more than 90 per cent low-temperature ground mass of hydrated silicates and carbonaceous matter, of grain-size less than $l \mu$, in which are embedded globular or rounded particles, varying in diameter from 5 to $50 \mu$, of magnetite, glass, olivine, troilite and other high-temperature minerals. I have interpreted ${ }^{4,5}$ these to be 'microchondri', that is, solidified 'fog' particles from an incandescent cloud, as often they reveal exsolution cores. In the 'type II' stones, the ground mass is reduced to about 60 per cent and larger chondrules, that is, 'raindrops', are present in addition to the 'microchondri'. Most of these are, however, of the 'bunch of grapes' type, and still reveal the original microchondrules. These apparently became compacted in a viscous or sticky state. Within 'type III' stones the ground mass is reduced to about 35 per cent, and the majority of the chondrules are 'fully coalesced'; the latter stones grade into the non-carbonaceous chondrites. The three hitherto known ureilites contain between 0.5 and 1 per cent carbon, and they consist of what appear to be squashed single crystals or granular aggregates (possibly of 'microchondrules') of silicates, with concentrations of carbonaceous matter and diamonds along the grain boundaries. The ten enstatite chondrites contain 3-10 per cent carbon + water + sulphur, and, roughly speaking, have a petrographical structure similar to the 'type III' carbonaceous stones or the ordinary chondrites. More detailed descriptions of the carbonrich groups of meteorites are to be found elsewhere $2,4,6,7$.

This article describes some recently observed examples of inhomogeneities, inclusions and interbrecciation, in meteoritic materials of which at least one sample belongs to the foregoing 'carbon-rich' groups.

\section{(1) Orgueil}

\section{Inclusions}

Inclusion $A$. In the course of preparation of material for analytical work during 1962 , I carefully separated some $3 \mathrm{~g}$ of the stone, obtained from a 20 -g crust-less fragment from the Museum in Paris. An unusually hard, angular inclusion was found, which on analysis revealed a rather higher percentage of troilite plates and magnetite microchondrules than was present in the rest of the stone; however, the petrographical structure remained within the range of the 'type I' stones. An essentially similar type of angular inclusion was observed in a thin section of the Orgueil meteorite ${ }^{8}$.

Inclusion $B$. I observed in a thin section of a roughsurfaces specimen of the Orgueil material from the Paris Museum an inclusion of about $1.5 \times 1.0 \mathrm{~mm}$ cross-section. This had an approximately globular habit (in fact, in outline, it had the shape of a jug) and in cross-section appeared to consist of some eight 'bunch-of-grapes'-type 